with Level I denoting strongly evidence-based statements and Level III denoting statements unsupported by evidence. In 2001, the level definitions were revised to denote the quality of specific study designs, and explicit guidance for synthesising bodies of evidence was developed. A third revision in 2006 tightened the criteria for Levels I and II, and created a Level IV to differentiate poor-quality studies from expert opinion. In 2011, the levels were revamped to be consistent with SIGN and GRADE methods.

Lessons The Academy has worked for years to make its evidence grading systems more rigorous. This has included using a levelbased system to clearly link PPP recommendations to evidence quality, revising the level definitions for greater clarity, and providing explicit direction on evidence synthesis.

\section{P224 ADAPTING GUIDELINES WITH CONFLICTING RECOMMENDATIONS: THE CASE OF MICROSCOPIC ASYMPTOMATIC MICROHEMATURIA}

J Whittaker, R Loo. Southern California Permanente Medical Group, Pasadena, USA

\section{0:1136/bmjas-2013-002293.214}

Background Existing clinical practice guidelines (CPGs) conflict on the best approach to the work-up of patients with asymptomatic microscopic hematuria who are at low risk of urinary tract cancer. Consequently, while adapting existing CPGs to a local context can be an attractive alternative to de novo development, when there are conflicting guidelines, disagreement with recommendations, or missing critical outcomes, potential time and resource gains are often minimised due to the need for additional evidence assessment and stakeholder consensus building activities.

Context To reconcile conflicting recommendations and address gaps in evaluation of critical outcomes, a Guideline Development Team (GDT) in a regional health care organisation used existing CPGs and systematic reviews, supplemented by additional evidence reviews, to develop recommendations for asymptomatic microscopic hematuria.

Description of Best Practice Based on review of evidence synthesised in external guidelines, supplemental de novo evidence reviews on critical outcomes, and risk assessment analysis of patient data, the GDT reached consensus on recommendations that differed from external guidelines, concluding that asymptomatic hematuria patients without risk factors were at sufficiently low-risk for urinary tract cancer to safely eliminate multiphase CT urograms from most urologic evaluations. Guidelines to reduce unnecessary CTs and radiation exposure in low-risk patients were developed, and knowledge transfer interventions aimed at the practitioner and healthcare system levels were implemented.

Lessons for Guideline Developers When adapting external guidelines with conflicting recommendations and gaps in critical outcomes, additional evidence searches, data analysis, and consensus building can negate anticipated gains in time and resources expected from guideline adaptation.

\section{P225 THE DEVELOPMENT OF EVIDENCE BASED GUIDELINES FOR OPIOID PAIN TREATMENT}

${ }^{1} \mathrm{~A}$ Effiong, ${ }^{1} \mathrm{~K}$ Hegmann, ${ }^{1} \mathrm{M}$ Thiese, ${ }^{2} \mathrm{C}$ Wolfkiel, ${ }^{2} \mathrm{~J}$ Ording, ${ }^{3} \mathrm{~J}$ Harris, ${ }^{1} \mathrm{~K}$ Schwei. ${ }^{1}$ Rocky Mountain Center for Occupational and Environmental Health, Salt Lake City, USA;
${ }^{2}$ ACOEM, Elk Grove Village, USA; ${ }^{3}$ The Permante Medical Group, San Rafael, United States

\section{0:1136/bmjqs-2013-002293.215}

Background Opioid analgesic-related deaths more than tripled in the US from 1999 through 2006. According to the CDC, 27,000 unintentional drug overdose deaths occurred in the United States in 2007; the most potent epidemic in the past 100 years.

Objectives To develop opioid guidelines to improve opioid pain treatment and reduce fatalities.

Methods A systematic literature search (including Google Scholar and Medline) was conducted. Randomised controlled trials (RCTs) were categorised into acute, subacute (1-3mo), chronic, and mixed chronicity. The quality of RCTs was determined using previously developed guideline scoring methods; low quality (3.5 or less), moderate quality (4.0-7.5), and high quality (8.0-11.0).

Results A total of 153 RCTs were identified; 11 acute, 2 subacute, 137 chronic, and 3 of mixed chronicity. Of the 11 acute pain RCTs, 1 was low quality, 6 moderate quality, and 4 high quality. Both subacute pain RCTs were moderate quality. Of the 137 chronic pain RCTs, 28 were low quality, 95 moderate quality, and 14 high quality. The RCTs with mixed chronicities were of moderate quality. All trials were under 6 months, with most under 4-weeks duration, precluding statements on long-term safety.

Discussion These opioid guidelines provide more informed recommendations for prescribing opioids for pain treatment with details to be presented.

Implications These guidelines may have considerable implications among prescribing health professionals.

\section{P226 A MULTIDISCIPLINARY APPROACH TO CREATING BEHAVIORAL HEALTH GUIDELINES: CHALLENGES OF ADHERING TO IOM STANDARDS}

R Halfond, L Bufka, H Kurtzman, D Galper, S Beattie. American Psychological Association, Washington, D.C., United States

\section{0:1136/bmjqs-2013-002293.216}

Background In 2010 our organisation began creating evidencebased clinical practice guidelines for behavioural health, focusing initially on depression, PTSD, and obesity, following emerging IOM standards.

Context Selected challenges in five areas: 1. Terminology. For many years, our organisation used guideline terminology that was inconsistent with the field. 2. Representation. Given the diverse types of professionals in behavioural health and the breadth/depth of each topic, attaining sufficiently diverse panel membership has been challenging. 3. Stakeholders. Obtaining the patient perspective has been challenging, particularly given the stigma and privacy concerns often associated with mental health. 4. Systematic Reviews. The high cost of developing de novo systematic reviews, especially for large scope topic areas, is limiting. 5. Education. Professionals have varying knowledge and lexicons for the process, requiring education, particularly surrounding non-financial conflicts of interest.

Description of Best Practice - Terminology- Implemented organisation-wide systemic change in lexicon via change in organisation policy and routine dissemination. - Representation- Used multi-step consensus nomination process to assemble 